\section{Non-coding RNAs and cancer: microRNAs and beyond}

\author{
Rachel Raia, George Adrian Calin \\ Department of Experimental \\ Therapeutics, University of Texas MD \\ Anderson Cancer Center, Houston, TX, USA
}

\section{Abstract}

Non-coding RNAs were previously thought to have little importance because they are not directly translated into a protein like their coding counterparts. However, it was recently found that non-coding RNAs do in fact have a much bigger role than previously thought. They are involved in cancer predisposition, development and progression. MicroRNAs, very short non-coding RNAs, are abnormally expressed in cancer and some harbor mutations that affect expression levels. MicroRNA alterations have been observed in all forms of cancer that have been researched to the current date. MicroRNAs are also located in cancer-associated genomic regions, which have been previously shown to affect gene expression leading to the activation or inhibition of cancer growth. Single-nucleotide polymorphisms within microRNAs can predispose someone to cancer. MicroRNAs have been shown to target both tumor suppressors, inhibiting cancer development, as well as oncogenes, stimulating cancer development. Some microRNAs can switch between these two functions and behave as a tumor suppressor at one time and an oncogene at another time. MicroRNAs can be used for diagnostic purposes as well as prognostic evaluations. Outside of microRNAs, ultraconserved genes, another group of non-coding RNAs, also express differently in cancer patients. Large intervening non-coding RNAs, specifically one termed HOTAIR, have been quantified in very high levels in cancer cells and have been implicated in metastasis. Further research into noncoding RNAs may allow for the development of therapies that will target non-coding RNAs creating better treatment options for cancer patients, improving their prognosis. This review discusses the most current discoveries about non-coding RNAs, revealing their associations with cancer.

\section{Introduction}

MicroRNAs are short non-coding RNAs, usually only about 20 nucleotides in length. They are formed from precursor microRNAs in the form of hairpin loop structures. ${ }^{1}$ An interesting characteristic of microRNAs is that they are highly conserved among orthologous species, indicating their importance in basic cellular processes. In fact, these short sequences are essential in many cellular processes, including cell-cycle regulation, stress response, development, differentiation, immunity, metabolism, apoptosis, aging and neuronal patterning. ${ }^{2,3}$ One mechanism of microRNA action is the influencing of mRNA cleavage resulting in mRNA degradation (Figure 1A). ${ }^{4}$ The study of microRNAs and their implications in cancer initiation, progression and dissemination have been continuously expanding as the science community continues to discover more about microRNAs and their effects on gene expression and subsequent cancer development. Effective therapies for various types of cancer may lie in how microRNAs stimulate or inhibit cell proliferation.

\section{All human cancers demonstrate microRNA alterations}

MicroRNAs play an important role in tumorigenesis. It has been observed that microRNA sequences are abnormally expressed in cancer. B-cell chronic lymphocytic leukemia (CLL) was the first type of cancer in which microRNA alterations were observed. ${ }^{5}$ Subsequently, varied expression of microRNAs between normal and cancer cells have been recognized in other types of cancer. In fact, microRNA alterations have been observed in every type of cancer that has been scientifically studied. ${ }^{6}$ In addition, genetic mechanisms can lead to overexpression, or on the contrary, suppression of microRNAs. ${ }^{7}$ An example is the down-regulation of let- 7 in lung and breast cancers and miR-15 and miR-16 in chronic lymphocytic leukemia. ${ }^{7}$ Also in lung and breast cancers, it has been observed that miR-17-92 over-expression leads to cancer growth. ${ }^{7}$ Also, mutations within microRNAs are more prevalent in cancer cells as compared to normal cells, which shows that these mutations have a role in tumorigenesis and the characteristic malignant phenotype of a cancer cell. ${ }^{6}$

Many different microRNAs have been shown to be involved in cancer and are, in most cases, located in areas of the gene that have been previously determined to be involved in cancer. MicroRNAs have been found in CAGRs (cancer-associated genomic regions). This is significant because CAGRs are established places where malignant mutations have been proven to occur. ${ }^{8}$ HOX genes, transcription factors that have been shown to cause carcinogenesis, are located in association with microRNAs. This provides further evidence of the importance of
Correspondence: MD, PhD, George A. Calin, University of Texas MD Anderson Cancer Center, Department of Experimental Therapeutics, Unit 0036, 1515 Holcombe Blvd., Houston, TX 77030, USA. Tel: +1.713.792-1202.

E-mail: gcalin@mdanderson.org

Key words: microRNAs, cancer, UCR, lincRNA, HOTAIR.

Acknowledgemnents: G.A.C. is supported as a Fellow at The University of Texas M.D. Anderson Research Trust, as a Fellow of The University of Texas System Regents Research Scholar and by the CLL Global Research Foundation. Work in $\mathrm{Dr}$ Calin's laboratory is supported in part by an NIH , by a DOD Breast Cancer Idea Award, by Developmental Research Awards in Breast Cancer, Leukemia and Brain SPOREs, by a CTT/3I-TD grant and by 2009 Seena Magowitz Pancreatic Cancer Action Network - AACR Pilot Grant.

Conflict of interest: the authors report no conflicts of interest.

Received for publication: 24 January 2011.

Revision received: 1 March 2011.

Accepted for publication: 4 March 2011.

This work is licensed under a Creative Commons Attribution 3.0 License (by-nc 3.0).

(C) Copyright R. Raia and G.A. Calin, 2011 Licensee PAGEPress, Italy

Journal of Nucleic Acids Investigation 2011; 2:e5 doi:10.4081/jnai.2011.e5

microRNAs in cancer proliferation. ${ }^{8}$ MiR-155, which is over-expressed in leukemia, is located within BIC, the B-cell integration cluster which has been implicated in faster rates of carcinongenesis. ${ }^{8}$ In addition, the presence of some microRNAs has been shown to decrease the risk and rate of cancer development. For example, miR-126 has decreased the growth of cancer cells and miR-335 has reduced the metastasis of cancer cells when both miR-126 and 335 were restored in the cancer cell environment after having had decreased expression. ${ }^{9}$ In essence, the restoration of these microRNAs reversed the progression of tumor cell growth and development.

\section{Alterations in microRNA might influence predisposition to developing cancer}

Variations in sequence that cause microRNAs to be abnormally expressed may provide a new method of determining cancer predisposition. ${ }^{2}$ From studies done on CLL, 
miR-16 has been shown to be involved in cancer predisposition. ${ }^{6}$ MiR-21 is over-expressed in many types of cancer. ${ }^{2}$ In addition, germline variants help to pinpoint which genes are involved in familial predisposition to cancer. ${ }^{10}$ For example, SNPs (single-nucleotide polymorphisms), which are point mutations found within microRNAs, have been observed to increase the risk of cancer when a patient's medical history shows genetic predisposition to developing cancer. ${ }^{10}$ SNPs that occupy microRNA binding sites may cause a change in protein function, thus increasing a person's chance of being predisposed to developing a certain type of cancer. ${ }^{11}$ A woman's risk of developing ovarian cancer has been shown to be positively correlated to SNPs in microRNA binding sites, whereas variant alleles of the same SNPs are inversely correlated with cancer risk. ${ }^{12}$ This means that only certain SNP alleles are associated with cancer risk, whereas others are present in the absence of cancer. MicroRNA binding site SNPs are not only involved in cancer predisposition, but may also have implications in determining diagnosis and prognosis for patients with cancer. ${ }^{11}$ Further study of microRNA signatures may allow for screening tests to be developed that would have the capability of determining whether or not someone is predisposed to having a specific type of cancer.

\section{microRNA can function as tumor suppressors, oncogenes or both}

MicroRNA genes that contribute to cancer development are called oncogenes, and those that inhibit cancer progression are called tumor suppressors. However, some microRNA genes are not exclusively classified as either oncogenes or tumor suppressors, but instead can have dual functions acting as either activators or suppressors depending on the type of cancer cell they express as well as microRNA alterations. ${ }^{13}$ An example of these microRNAs with both properties is miR-17-19b-1 which is an oncogene but can also act as a tumor suppressor if it targets E2F1, a gene that encodes the E2F1 transcription factor and controls tumor suppressor proteins and regulates the cell cycle. ${ }^{8}$ Another example is miR-181a which can act as an oncogene or a tumor suppressor depending on what kind of cell it is found in. ${ }^{8}$ In CLL, miR-181a/b and miR-29a/b have been proven to be tumor suppressors, directly targeting the TCL1 oncogene. ${ }^{2}$

Some microRNAs act only as tumor suppressors or oncogenes, not both. In colorectal cancer, miR-17-92 has been found to be an onco-
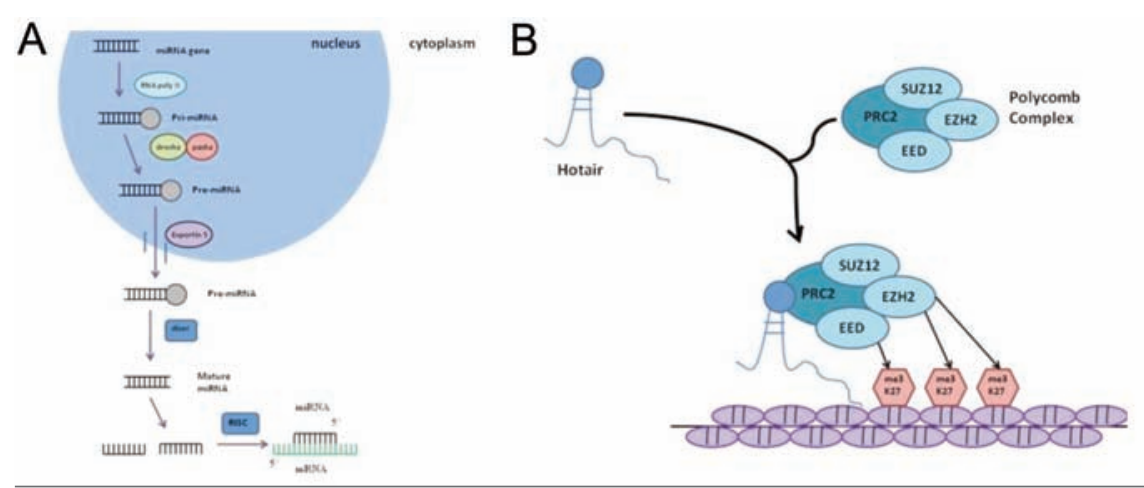

Figure 1. Mechanism of action of microRNAs and lincRNAs. (A) RNA polymerase II transcribes a microRNA gene to form a pri-miRNA. Drosha and pasha create a pre-miRNA from the pri-miRNA. Exportin 5 moves the pre-miRNA out of the nucleus and into the cytoplasm. Dicer produces a mature miRNA. Then, RISC processes the mature miRNA. One strand is chosen and messenger RNA binds it complementarily to the selected miRNA. Target mRNA is produced. (B) HOTAIR attaches to the polycomb complex. Methylation occurs when the HOTAIR with the PRC2 attaches to me3k27.

gene by promoting growth of cancer cells and inhibiting apoptosis, defined as programmed cell death. ${ }^{5}$ The tumor suppressor gene, TP53, which controls the transcription of miR-34, has been shown to regulate senescence, naturally occurring biological aging, as well as apoptosis, showing that this tumor suppressor plays a role in upstream regulation. ${ }^{5}$

Not only can the presence of microRNAs cause or inhibit cancer development, but their absence can also cause similar effects. For example, if tumor suppressors are silenced, such as in the let7 family, the absence of the expression of these microRNAs may cause cancer cells to proliferate at a much higher rate than if those tumor suppressors had been expressed normally. ${ }^{14}$ In contrast, the silencing of oncogenes, such as miR-155, may inhibit the proliferation of cancer cells, and thus, slow the development of cancer, presenting potential targets for treatment therapies.

\section{Diagnostic tools for cancer include microRNA profiling}

Diagnostic tools for cancer have been developed that identify microRNA signatures, meaning lists of microRNAs differentially expressed or associated with specific clinical parameters. These tools include bead-based flow cytometry, isolation of microRNA clones (microRNA amplification profiling) and oligonucleotide microRNA microarray chips. ${ }^{8}$ In addition, microarray analysis of a blood sample can reveal microRNA fingerprints present in blood, which may be used to diagnose ovarian cancer because these profiles are disease-specific, eliminating the possibility of mistakenly diagnosing a patient with a differ- ent type of cancer. ${ }^{15}$ Using PCR analysis, microRNAs can be used as biomarkers for diagnosing cancer found in a patient's blood serum or plasma. ${ }^{16}$ Blood testing provides a relatively inexpensive, simple and fast method of diagnostic testing. Screening for various microRNA expression levels in order to diagnose a potential cancer patient is currently happening and will become more prevalent as more knowledge about microRNAs becomes available, ensuring accurate diagnoses. In addition, outside of cancer, microRNAs as diagnostic markers for other types of disease, including heart disease and immune disorders, are currently being researched. For example, microRNAs are down-regulated in patients with cardiomyopathies. ${ }^{9}$ Specifically, miR-1 and miR-133 are inversely related to hypertrophy; when these microRNAs are downregulated, hypertrophy is more likely to occur and when the same microRNAs are up-regulated, hypertrophy is inhibited. ${ }^{9}$

\section{microRNA profiling can be uti- lized to determine cancer prog- nosis}

MicroRNA expression has been shown to forecast the clinical progression of disease as shown specifically in CLL pathogenesis. ${ }^{2}$ In CLL, the oncogene TCL1 is a marker for poor prognosis because it is directly correlated to ZAP-70 (zeta-associated protein) expression. ${ }^{2}$ CLL patients with genetic deletions at locations $17 p$ and $11 q$ have been shown to have more progressive disease, along with a poorer prognosis, as compared to patients without these deletions. ${ }^{2}$ In a recent publication, it 
was shown that patients with $13 q$ deletions, associated with increased expression of both miR-15a and miR-16, had increased TP53 expression. ${ }^{17}$ In the same paper, decreased levels of ZAP70 were shown to be correlated with increased survival. ${ }^{17}$ Deletions and other genomic aberrations leading to cancer gene expression have proven to be accurate predictors of disease progression with deletions usually representing more aggressive cancer forms. More specifically, miR-223 and miR-29c are expressed at very low amounts in patients who are predicted to have a shorter survival time (poor prognosis). ${ }^{2}$ MiR-155 has also been shown to be highly expressed in poor prognosis cases. ${ }^{8}$ Acute myeloid leukemia (AML) patients who present with increased expression of miR-191 have been shown to have a reduced survival time. ${ }^{8}$ Also, serum microRNAs may influence biological processes and disease development at additional locations in the body, suggesting microRNA involvement in metastasis. ${ }^{18}$ By researching how microRNA expression is varied, more prognostic tools may be discovered so that patient survival time may be more accurately determined. Also, better therapeutic methods for cancer treatment and inhibition of tumor growth may lie within microRNA expression. The pharmaceutical industry is already investigating microRNAs that activate or inhibit metastasis as well as miRSNPs that affect gene expression in order to evaluate whether or not these microRNAs may be potential targets for new drug therapies. ${ }^{19}$

\section{Ultraconserved regions are also altered in cancerous cells}

Ultraconserved regions (UCRs) are strictly conserved, non-coding sequences that are the same among orthologous species as well as among individuals within the same species. These ultraconserved regions transcribe ultraconserved genes. ${ }^{6}$ It has been hypothesized that microRNAs may regulate the expression of ultraconserved regions in various cancers including colorectal cancer and CLL. ${ }^{5}$ There are some UCRs that have been specifically identified as playing a role in cancer. For example, UC.73A is an oncogene that promotes colon cancer. When expression is decreased, cancer cell growth and proliferation is inhibited. ${ }^{5}$ Also, UC.159 has deletions more often in cancer patients as compared to normal controls. ${ }^{6}$ In CLL, UC.73 has also been shown to be expressed higher in cancer samples as compared to normal samples, and when repressed, colon cancer cell proliferation was inhibited. ${ }^{9}$ Usually, deviation from the normal popula-

Table 1. Comparison between several categories of ncRNAs.

\begin{tabular}{llll}
\hline Property & miRNAs & UCGs & lincRNAs \\
Length & (T-UCRs) & & \\
Binding protein & $20-21 \mathrm{nt}$ & $>200 \mathrm{nt}$ & $>200 \mathrm{nt}$ \\
\hline $\begin{array}{l}\text { Number of human genes } \\
\text { Expression patterns }\end{array}$ & Argonaute family & unknown & PRC2 \\
\hline Biogenesis & Most cell types & Most cell types & Most cell types \\
Conservation & Dicer dependent & Unknown & Unknown \\
\hline Function & High & Highest & Low \\
& Post-transcriptional & Possibly gene regulation & Gene regulation \\
\hline miRNAs, microRNAs; UCGs, ultraconserved genes; T-UCRs, transcribed ultraconserved regions; lincRNAs, long non-coding RNAs; PRC2, poly- \\
comb repressive complex 2.
\end{tabular}

tion's ultraconserved sequence is observed in people who have some type of abnormal clinical condition, including cancer. ${ }^{6}$ Oncology patients have more ultraconserved region mutations than those without cancer. ${ }^{6}$ Transcribed ultraconserved regions (T-UCRs) are usually found in cancer-associated genomic regions as well as in fragile sites, meaning that these T-UCRs may play a role in the development of cancer, and could therefore be a target for therapy. ${ }^{9}$ As of January 2011, no therapies have been discovered that use UCGs as therapeutic targets in cancer. ${ }^{9}$ UCR sequences that differ from the norm have been proven to contribute to the malignancies brought on by cancer cells. $^{20}$

\section{Hotair is a lincRNA important in metastasis}

Not only short non-coding RNAs are implicated in cancer (See Table 1 for ncRNA comparisons). It has been recently discovered that large intervening non-coding RNAs (lincRNAs), specifically one in this class called HOTAIR (Figure 1B), are involved in cancer metastasis. ${ }^{21}$ These lincRNAs are referred to as long non-coding RNAs because they have many more nucleotides in their sequence as compared to short ncRNAs. In fact, long ncRNAs are usually composed of several hundred nucleotides, as opposed to the twenty or so nucleotides that comprise short ncRNAs. The HOTAIR gene is termed from HOX Antisense Intergenic RNA. ${ }^{21}$ HOTAIR binds PRC2, the Polycomb repressive complex. PRC2 functions as a silencer of tumor suppressor genes, thus promoting cancer progression. ${ }^{22}$ Also, it has been found that HOTAIR may cause the PRC2 complex to change genomic locations. ${ }^{22}$ HOTAIR sends the PRC2 complex to target genes to silence tumor suppressors, eliminating any inhibition of cancer progression and metastasis. ${ }^{22}$ HOTAIR is extremely overexpressed in metastasized tumor cells as compared to normal cells. These things combined create a mechanism that could potentially explain the significance of highly increased expression of HOTAIR in metastasized tumors, thus explaining the association between HOTAIR and cancer metastasis. HOTAIR has been found to be over-expressed in metastasized tumors of both breast and lung cancers. ${ }^{22}$ Metastasized tumors that express HOTAIR and PRC2 may be sensitive to drugs that inhibit these two components. If chemotherapy medications are developed that target lincRNAs and, as a result, HOTAIR and PRC2, these treatments may be able to inhibit the growth and metastasis of breast and lung cancers cells as well as other types of cancer that involve lincRNAs. ${ }^{22}$

\section{Conclusions}

Discoveries about microRNAs and their critical roles as oncogenes and tumor suppressors are being made every day. The initial link between microRNAs and CLL, discovered in 2002 , opened new topics of research with significant clinical applications. The close associations between expression levels of various microRNAs and the development of cancer have shown that microRNAs are related to cancer predisposition and tumor development, proliferation and inhibition. The high correlations between microRNA expression and other known genetic factors that have been previously determined to be associated with cancer, such as cancer-associated genomic regions (CAGRs) and ultra conserved regions (UCRs), linked these genes to cancer genomics and gene evolution. Because the study of the correlations between microRNAs and cancer is a new field, many areas still need to be thoroughly researched before any conclusions can be made as to definitive causes and effects 
between microRNA expression and the regulation of cancer proliferation and growth inhibition. As research about microRNAs and their implications in cancer progresses, scientists hope to use microRNAs to develop further advanced technology, making earlier cancer diagnoses and more accurate prognoses possible. In addition, using knowledge about microRNAs, researchers hope to develop more efficient therapies for cancer patients.

\section{References}

1. Ambros, V. The functions of animal microRNAs. Nature 2004;431:350-5.

2. Calin GA, Croce CM. Chronic lymphocytic leukemia: interplay between noncoding RNAs and protein-coding genes. Blood 2009;114:4761-70.

3. Leung AKL, Sharp PA. MicroRNA functions in stress responses. Molecular Cell 2010;40:205-15.

4. Esquela-Kerscher A, Slack FJ. Oncomirs microRNAs with a role in cancer. Nat Rev Cancer 2006;6:259-69.

5. Rossi S, Kopetz S, Davuluri R et al. MicroRNAs, ultraconserved genes and colorectal cancers. Int $\mathrm{J}$ Biochem Cell Biol 2010;42:1291-7.

6. Wojcik SE, Rossi S, Shimizu M, et al. Noncoding RNA sequence variations in human chronic lymphocytic leukemia and colorectal cancer. Carcinogenesis 2009;31:208-15.
7. Taby R, Issa JPJ. Cancer Epigenetics. CA Cancer J Clin 2010;60:376-92.

8. Fabbri M, Garzon R, Andreeff M, et al. MicroRNAs and noncoding RNAs in hematological malignancies: molecular, clinical and therapeutic implications. Leukemia 2008;22:1095-105.

9. Edwards JK, Pasqualini R, Arap W, Calin GA. MicroRNAs and ultraconserved genes as diagnostic markers and therapeutic targets in cancer and cardiovascular diseases. J Cardiovasc Transl Res 2010;3:2719.

10. Van Kessel AG. Review of The 'omics' of cancer. Canc Genet Cytogenet 2010;203: $37-42$.

11. Pelletier C, Weidhaas JB. MicroRNA binding site polymorphisms as biomarkers of cancer risk. Expert Rev Mol Diagn 2010;10:817-29.

12. Liang D, Meyer L, Chang DW, et al. Genetic variants in microRNA biosynthesis pathways and binding sites modify ovarian cancer risk, survival, and treatment response. Cancer Res 2010;70:9765-76.

13. Rossi S, Sevignani C, Nnadi SC, et al. Cancer-associated genomic regions (CAGRs) and noncoding RNAs: bioinformatics and therapeutic implications. Mamm Genome 2008;19:526-40.

14. Scaruffi P, Stigliani S, Moretti S, et al. Transcribed-ultra conserved region expression is associated with outcome in high-risk neuroblastoma. BMC Cancer. 2009;9:441.
15. Hausler SFM, Keller A, Chandran PA, et al. Whole blood-derived miRNA profiles as potential new tools for ovarian cancer screening. Br J Cancer 2010;103:693-700.

16. Wittman J, Jack HM. Serum microRNAs as powerful cancer biomarkers. Biochim Biophys Acta 2010;1806:200-7.

17. Fabbri M, Bottoni A, Shimizu M, et al. Association of a microRNA/TP53 feedback circuitry with pathogenesis and outcome of B-cell chronic lymphocytic leukemia. JAMA 2011;305:59-67.

18. Cortez MA, Calin GA. MicroRNA identification in plasma and serum: a new tool to diagnose and monitor diseases. Expert Opin Biol Ther 2009;9:703-711.

19. Nagpal JK, Rani R, Trink B, Saini KS. Targeting miRNAs for drug discovery: a new paradigm. Curr Mol Med 2010;10:50310.

20. Taccioli C, Fabbri E, Visone $\mathrm{R}$, et al. UCbase \& miRfunc: a database of ultraconserved sequences and microRNA function. Nucleic Acids Res 2009;37:D41-8.

21. Rinn JL, Kertesz M, Wang JK, et al. Functional demarcation of active and silent chromatin domains in human HOX loci by non-coding RNAs. Cell 2007;129:1311-23.

22. Gupta RA, Shah N, Wang KC, et al. Long non-coding RNA HOTAIR reprograms chromatin state to promote cancer metastasis. Nature 2010;464:1071-6. 